\title{
A Novel Approach for the Detection of Road Speed Bumps using Accelerometer Sensor
}

\author{
Bassam Al-Shargabi ${ }^{1}$, Majid Hassan ${ }^{2}$, Thamer Al-Rousan ${ }^{3}$ \\ ${ }^{1}$ Faculty of Information Technology, Middle East University, Amman, Jordan \\ ${ }^{2}$ King Hussein School of Computing Sciences, Princess Sumaya University for Technology, Amman, Jordan \\ ${ }^{3}$ Faculty of Information Technology, Isra University, Amman, Jordan
}

\begin{abstract}
Smart phones and tablets have turned into an indispensable piece in our society. Accordingly, these devices are equipped with many sensors that can be exploited in many applications such as monitoring road surface condition. It becomes important to find an optimal method for detecting road speed bumps and potholes in order to monitor the road conditions and to alert other moving vehicles in order to slow their speed and avoid any damage and experience safe and comfortable driving. Moreover, data collected by smartphones about road conditions can help city authorities to repair and maintain the roads. The aim of this paper is to introduce a model for detecting road speed bumps and potholes using an Android application based on accelerometer sensor. This application uses data gathered by accelerometer sensor that was collected from various roads in order to determine the accuracy of this proposed approach. The proposed model was implemented as an Android application as a proof of concept and it was tested to check its accuracy.
\end{abstract}

Keywords - smartphone, accelerometer sensor, road bump detection, mobile application, internet of things

DOI: 10.18421/TEM92-07

https://doi.org/10.18421/TEM92-07

Corresponding author: Jordan Bassam Al-Shargabi, Faculty of Information Technology, Middle East University, Amman, Jordan.

Email: bshargabi@meu.edu.jo

Received: 27 September 2019.

Revised: 07 March 2020.

Accepted: 14 March 2020.

Published: 27 May 2020.

(cc)BY-NC-ND (C) 2020 Bassam Al-Shargabi, Majid Hassan, Thamer Al-Rousan; published by UIKTEN. This work is licensed under the Creative Commons AttributionNonCommercial-NoDerivs 3.0 License.

The article is published with Open Access at www.temjournal.com

\section{Introduction}

With the widespread of Internet of Things (IoT) technologies many applications and services emerged through utilizing the concept of IoT. As for the term, IoT is refers to the integration of any computing devices, mechanical and digital equipment, and objects with the capability to transfer and generate data across a network without the need for human or computer interactions. Another way to look at the IoT as group of objects, devices, and sensors that are interconnected and communicated to offer a value added services to consumer and enterprises [1].

The magic behind the IoT has already enabled an easy interaction and access with all kinds of objects or sensors that can be used in a wide variety of applications such as health care, home automation, connected roadways, smart cities and so on. Such devices are wearable devices, mobile phones that can be integrated to generate a vast amount of data that will lead to automation of any industry using the IoT technologies [2].

The latest state of the art in the design of the IoT technologies are stimulating for the development of new smart systems that will boost the competitiveness of SMEs and make people's daily life easier. The IoT has been exploited in building application for smart cities, where the current dilemma of the Smart City is the traffic monitoring systems. The traffic can be monitored through cameras installed on streets or through a cheap and efficient method such as the GPS systems that are installed in mobile phones or in modern vehicles [3],[4],[5]. The gathered data can be used to monitor and predict traffic congestion and road surface condition. Nevertheless, another application of the IoT in smart cities where city street conditions must be monitored endlessly to improve the transportation systems in terms of safe and comfort driveling. As the IoT enabled technologies can be enabled to detect anomalies in road surface conditions in terms of holes, bumps. 
The IoT enabled applications on mobile phones can help to harvest the internal sensors existing on mobile phones that can be exploited to use these sensors to perceive the orientation of the mobile phones to detect and help monitor the condition of roads. As a matter of fact, these embedded sensors in mobile phones can help monitor the road conditions such as poor construction quality, traffics, poor drainage, the roads temperature that may cause crakes and potholes on streets [6]. Therefore, mobile phones with their embedded sensors such as accelerometer sensor can be used to collect data about the condition of the roads such holes and bumps. The accelerometer sensor can be exploited to detect the road speed bumps and the GPS can be exploited to keep track of bumps' locations as when the vehicle goes through a speed bump or hole the mobile phones can sense it and determine bumps or holes' location. Accordingly, the gathered data can used by authorities to monitor street condition in order to repair and maintain the roads, also these data can be used to notify other vehicles from these bumps and holes in order for these vehicles either to slow their speed or avoid bumps and holes especially when we are talking about the auto-driving vehicles where such applications can have major impact on this industry.

Many approaches were developed for authorities in smart cities to find cost effective way to monitor the road surface with a greater and efficient methods to detect holes and speed bumps. As a matter of fact, city authorities demand a real time data collection method to monitor road conditions. There are many traditional approaches to monitor road condition such as $3 \mathrm{D}$ reconstruction, vibration, and vision-based [7],[8],[9]. The 3D reconstruction method focuses on 3D laser scanning to generate models for current road condition and compare it to the base model of roads to find any changes in roads condition or find any holes and speed bumps [10]. The second approach is vision-based approach, where it focuses on capturing images and videos of the roads through exploiting images taken by a camera/video system facing the roads from a moving vehicle [11]. Thereafter, these images and videos are processed in order to extract features, such as texture extraction, and compare these images with older images of the road surface in order to make comparison to find any anomalies on the road surface distress features, including road holes, speed bumps, and cracks [12]. The third approach is based on installing a sensor such as accelerometer sensor within moving vehicles. This sensor can detect any changes in roads based on the rate of vibration that occurs from the moving vehicles' vibrations. Hypothetically, when a vehicle goes through any road and faces speed bumps, holes, and cracks, it will vibrate more than when it moves over anomaly free road as we used this approach as part of the proposed model in this paper.

Furthermore, approaches and methods also have been proposed in literature to deal with the problem of detecting speed bumps on roads, as many researchers exploited the images processing techniques to detect speed bumps using Disparity approaches. some of these methods rely of detecting bumps and other obstacles on roads using a monocular IR camera, where this method of use compensates shadows on the road using Open Source Computer Vision (OpenCV) [7]. Another real-time system to detect the speed bumps via the analysis of the images collected from the roads and the use of Gaussian filter and computer vision to accurately detect speed bumps. This approach relies on having a huge number of images collected previously for the roads to compare it with the new images in order to detect speed bumps but this process requires more computing power in order accurately predict the speed bumps. [12].

With the widespread of use of mobile phones along with its sensing capabilities, it becomes important to find an efficient method in terms of accuracy and cost for detecting road speed bumps and holes in order to monitor the road conditions and to alert other moving vehicles to slow down their speed and avoid any damage and experience safe and comfortable driving. In addition, consideration must be taken into account when using sensing capabilities in mobile phones since these embedded sensors may differ based on many aspects such as the sensor's features and the types of mobile phones' position, vehicle's suspension system, and speed [6], which was covered and solved by the proposed model in this paper.

The rest of the paper is organized as follows: Section 2 discusses the related works to the proposed model that is based on finding an efficient method for detecting road speed bumps. Section 3 describes the proposed model while in section 4 are explained the experimental results that were conducted through developing an Android mobile application as a proof of the concept of the proposed model and it was tested to check its accuracy. Finally, conclusion was drawn up in section 5 .

\section{Related Work}

Many studies in literature have been conducted with the aim of detecting road speed bumps with each research paper trying to come up with solution to this problem in timely and cost effective manner. The researches in this area differ based to how they look at the issue from their own perspectives. Some of the researches focus on how data were collected while the others focus on how gathered data is 
processed in order to detect road speed bumps and holes.

An approach was presented in [14], where they utilized an Android mobile phone with a fixed direction to gather the acceleration data of any moving vehicles. Their data accumulation was done at a recurrence rate of $100 \mathrm{~Hz}$. The collected data was uploaded to a PC, where the data records were then checked to choose just those road segments to provide a complete data sets, where these data were used to classify roads into four classes based on road quality in term of speed bumps, cracks and holes.

Another approach presented in [15], is predominantly centered around the building part of their road surface checking framework. Their model was implemented on an Android application which assembles data from an accelerometer and GPS sensors. The sensors were kept running at the most elevated accessible recurrence. Since the GPS working recurrence was around $1 \mathrm{~Hz}$, about one sample of data was gathered each second. An examination was likewise completed, where the data accumulation application was introduced on two Motorola Moto $\mathrm{G}$ mobile phones, which were then put inside two public buses.

Tablet based approaches were utilized in [16] to gather data of various roads abnormalities. Three distinctive mounting arrangements in the vehicle were tried: totally fixed, mostly fixed (vertical axis free) and totally free. Moreover, they portrayed a procedure of reorienting the vertical speeding up utilizing two of the three Euler angles, explicitly the move edge $\alpha$ and the pitch angle $\beta$. The reorientation method depends on the way that when the vehicle and the tablets are stationary, where the main issue enlisted by the accelerometer to be dealt with is the increasing of speed of the moving vehicle because of gravity where such issue were handled by the proposed model in paper.

Moreover, an examination of the contrast between the arrangement of a roads peculiarities discovery and identification system in a "molded" and a certifiable setup is presented in [17], where the system performed more awful in contrast with the "adapted" setup. It additionally shows that their system was examined on training datasets; through the utilization of the PCA methods; alongside the examination of the inconsistency in the collected data through utilizing the standard deviation to validate the data used for training and testing in their system.

An investigation for road surface checking using mobile phones outfitted with GPS and inertial sensors: accelerometer and gyroscope was presented in [18]. In their investigation they portrayed directed examinations with data from time domain, frequency domain, and wavelet transformation where they used a strategy to decrease the impacts of speed, slants, and floats from sensor signals. The authors also used a varying data labeling method where their systems named RoADS, executes wavelet decomposition analysis for signal preparing of inertial sensor signals and Support Vector Machine (SVM) for oddity discovery and classification of road surface. Not to mention that labeling the road surface condition accurately is a difficult task, where this problem was tackled in [19],[20] by developing an android application called Road Data Collector that automates data collection and labeling, where data also was collected based on accelerometer sensor and gyroscope to derive more accurate road quality prediction.

An approach that includes the utilization of a multivariate genetic algorithm (GALGO) for road irregularities identification dependent on estimated data from a gyro, an accelerometer also, GPS sensor associated with an IoT gadget was proposed in [21]. The collected data gathered from the sensors were collected at regular intervals, where this data described by measurable parameters that can portrayed the entire datasets were extricated and utilized in preparing a genetic algorithm based machine learning system to develop a model for detection of the road speed bumps. A total of around 14,090 data sets were gathered during a drive test with more than ten unique laps at a speed of $20 \mathrm{~km} / \mathrm{hr}$. Measurable parameters, for example, the mean, fluctuation, skewness, kurtosis, standard deviation, dynamic range and most extreme signal sufficiency esteems was utilized to extract the feature to be used for machine learning algorithm. An agent model was chosen dependent on the frequency of appearance of an element in the created multivariate models utilizing a forward choice and in reverse end procedure. The authors used $70 \%$ of the datasets for training while, $30 \%$ were utilized for testing and approval. However, their approach proved that it is computationally unpredictable as it requires the utilization of such a large number of factual highlights to be extracted for the machine learning. Besides, this approach can't recognize other oddity, for example, potholes, Where this problem in the street roads is the main cause of fatal accidents in most developing countries due to the lack of warning signs.

A method in [22], for identifying and informing driver for the forthcoming un-checked and stamped road speed bumps, progressively utilizes deep learning methods and stereo-vision approaches. The authors have utilized NVIDIA GPU and Stereolabs ZED Stereo camera equipment. The vehicle can be controlled to avoid road speed bumps at certain rate even if that vehicle was driven by driver or autodrived where an alert of road speed bumps occurs so as not to make any sort of uneasiness the travelers 
just as harm to the vehicle[23], [13]. As in this paper we used the feature in the proposed model to permit threshold instated of using a certain rate.

\section{Proposed Model}

The purpose of the proposed model is to detect the road speed bumps and potholes by a mobile application through utilizing the Accelerometer and the GPS sensors embedded within the mobile phone to alert other vehicles and city authorities to keep track of the road conditions as illustrated in Figure 1.

The proposed model starts by gathering data based on Accelerometer reading values from mobile application within moving vehicles. These readings must be pre-processed and converted into a clean and organized data sets prior to analysis. As the sensors' row data set needed to be smoothed along with filtering the noise.

The noise filtering technique we used is based on noise-threshold to enable the mobile application to ignore the results caused by the phone-shaking as they should not be considered as bumps. In the proposed model, the noise-threshold value helps to capture only outputs resulting by true speed bumps and ignoring false outputs caused by small variations to eliminate the shakes of the vehicle or mobile phone without bumps or holes. After removing noise readings of accelerometers, for each sequence of accelerometer reading values if the readings are steady that means we are seeking to find beginning of a speed bumps. The none zero values indicates a detection of speed bumps, if there is a possibility of a continuous speed bumps then we are trying to determine if that speed bump ends. Once the start of speed bump was detected the Mobile application assigned statrting point for that bump along with time-stamp and keeps listening to and calculating for the next consecutive reads which we call it a "Transformed Y-Bump Factor" (TYBF). As long as this value is still positive, the application assumes we are still passing over a current bump till the value turns again to zero, then the application assigned that the current bump ended. When a bump is starting to occur, the value of TYBF changes from zero to a positive value. The amount of this value could vary according to the sharpness of the bump. For acute bumps, or holes, the value tends to be larger. For example, when we are going through speed bumps and the accelerometers' readings were positive and the time duration for detecting that bump was 2.883 seconds and the distance of this bumps was $.23 \mathrm{~m}$.

The speed bumps often consists of two or more consecutive irregular up/down bends. While crossing over a speed bump, the vehicle could inverse from up bend to down bend or from down to up bend. This causes the accelerometer to pick a zero change while monitoring the $\mathrm{Y}$ axis changes over the speed bump. Therefore, a typical bump yields consecutive positive values of the TYBF with some zero values occurring between the positive values. In this case, to solve the problem of continuity of speed bumps, In the proposed model, we dealt with these zero values as not real signs of ending the current bump. Accordingly, those readings will not be used to point to the end of speed bumps as the zero TYBF was detected. As long as the zero reading values that are repeated for certain amount of times during bumps detection will be ignored for a pre-specified constant number of times before assigning that the occurring bumps is ended. This pre-specified number is a constant number calculated by trial and error during the testing of the proposed model for the base algorithm. the pre-specified number is tuned to be reasonable for any value between 10 and 20 sequences for accelerometer reading values.

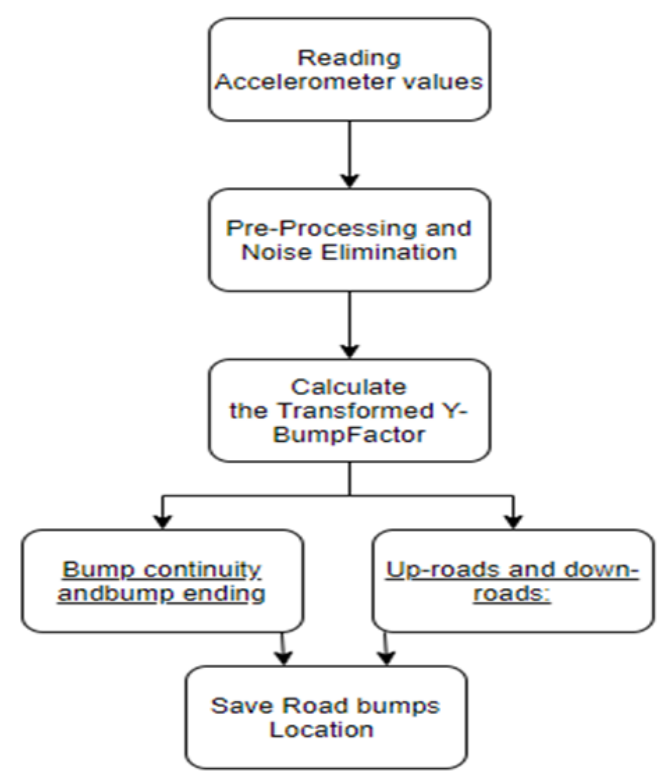

Figure 1. Proposed Model

In the proposed model, we tackled the issue of accelerometer reading values when up-roads and down-roads occurs, as they occur when $\mathrm{Y}$ axles reading of accelerometer values continuously increase or decrease. In the proposed model, we have to realize that most of the roads are not fully horizontal with zero changes in the $\mathrm{Y}$ axis along all the way even if the road seems to be like that. We intend to detect the road Slope-Effect (SE) by first calculating the result of dividing the average changes of Y-axis "Delta Y-Average" (DAvY) by average changes of $\mathrm{X}$-axis "Delta X-Average" (DAvX) of the continuous readings of accelerometer which can be calculated by equation 1 as seen below:

$$
\text { Slope-Effect }\left(S E_{n)}=D A v Y_{n} / D A v X_{n}\right.
$$


where $\mathrm{n}$ represents the number occurrences of changes in accelerometer reading of $\mathrm{Y}$-axis and $\mathrm{X}$ axis values. Second, we calculate the average of some consecutive occurrences to find what we called slope-effect-refinement-factor, which can be determined by equation 2 as seen below:

$$
\begin{aligned}
& \text { slope-effect-refinement-factor } k=\text { average } \\
& \left(S E_{1}, S E_{2}, S E_{3,}, \ldots, S E_{k}\right)
\end{aligned}
$$

where $\mathrm{k}$ represents the number of accelerometer reading that occurs during a specific time. In the proposed model, we have been convinced to perform the calculation of slope-effect-refinement-factor in regular periods basis which has been adjusted to be between 10 milliseconds up to 30 milliseconds. Finally, we involved the value of the calculated slope-effect-refinement-factor by adding it to the "noise-factor" base constant value each time we calculate it. By adding the slope-effect-refinementfactor value to the "noise-factor" we then actually have reduced the slope-effect of the road and taking into account to adjust the "noise factor" according to the changes in the road nature.

\section{Experimental Result and Discussion}

This section provides a full description of the experiments conducted to demonstrate the accuracy of the proposed model for road bump detection.

\subsection{Data Gathering}

Dataset gathering stage is the most significant one; since it is aimed for collecting data for road condition. The data gathering procedure has been collected for 2 hours using Huawei Y9 2019 mobile phone that was put inside a Toyota Prius vehicle. The dataset gathered through utilizing the accelerometer and gyrator sensors within Huawei Y9 20119 mobile phone. The sensors accumulated data along the vehicle way. The gathered data were put away locally inside the mobile phone memory. Additionally, the GPS directions of the physically stamped street bumps points are kept inside the memory.

We have designed an application for collecting the dataset which is not dedicated only for Huawei Y9 20119 , it can be configured to be used within any mobile phone. As mentioned earlier, the data gathering starts by activating the mobile phone accelerometer and gyroscope sensors with setting a timer to start receiving the readings of the sensors. Once data collection finished, the data exported into excel sheet in the mobile phone memory, a ample of the data gathered of accelerometers as an array of ( $\mathrm{X}$ and $\mathrm{Y}$ ) is shown in Table1.
Table 1. Sample of accelerometers readings

\begin{tabular}{|c|c|}
\hline $\mathbf{x}$ & $\mathbf{y}$ \\
\hline 2 & 2 \\
\hline 2 & 4 \\
\hline 2 & 6 \\
\hline 2 & 4 \\
\hline 2 & 5 \\
\hline 2 & 3 \\
\hline 2 & 7 \\
\hline 2 & 4 \\
\hline 2 & 6 \\
\hline 2 & 5 \\
\hline 2 & 5 \\
\hline 2 & 6 \\
\hline 2 & 4 \\
\hline 2 & 5 \\
\hline 2 & 3 \\
\hline 2 & 4 \\
\hline 2 & 6 \\
\hline 2 & 4 \\
\hline 2 & 7 \\
\hline 2 & 10 \\
\hline 2 & 14 \\
\hline
\end{tabular}

\subsection{Data processing and Normalization}

The pre-processing of gathered data consists of many steps in order to deal with data noise such as car shaking (among others). Therefore, for each accelerometer the reading instance is joined with other calculated values on $\mathrm{Y}$ values that yield into other arrays as follows:

\section{Y-Average of each Y read:}

Calculating the "Y-Average" (AvY), which is the average of $\mathrm{Y}$ gained from the previous accelerometer read and the current read of "Y"

2. Delta Y-Average for each Y read:

Calculating the "Delta Y-Average" (DAvY), which is the difference between "Y-Average" calculated for $\mathrm{Y}$ in the previous accelerometer read and the current calculated "Y-Average" (as per step 1)

3. X-Average of each $X$ read:

Calculating the "X-Average" $(A v X)$, which is the average of $X$ gained from the previous accelerometer read and the current read of "X".

4. Delta X-Average for each X read:

Calculating the "Delta X-Average" $(D A v X)$, which is the difference between "X-Average" calculated for $\mathrm{X}$ in the previous accelerometer read and the current calculated "X-Average" (as per step 3)

5. Noise-Free Delta Y-Average (Y) for each Y read: We calculated "Noise-Free Delta Y-Average" $(N F D A v Y)$ which can be gained by subtracting the constant value of "Noise-Threshold-Value" from the absolute value of current "Delta Y-Average". Noise-Threshold-Value was set to 2. Thereafter, in order to measure the changes of $\mathrm{Y}$-axis reading, we calculated the "Transformed Y-Bump Factor" (TYBF), which is the maximum of "Noise-Free Delta Y-Average" the current Y read and zero as shown in Table 2. 
Table 2. Dataset processing result

\begin{tabular}{|c|c|c|c|c|c|}
\hline DeltaY & Abs DeltaY & Avg deltaY & Avg Y & Delta AvgY & Transformed y-bump factor \\
\hline 2 & 2 & 2 & 3 & 1 & 0 \\
\hline 3 & 3 & 2.5 & 5.5 & 2.5 & 0 \\
\hline 1 & 1 & 1.75 & 7.5 & 2 & 0 \\
\hline 2 & 2 & 1.875 & 9 & 1.5 & 0 \\
\hline 3 & 3 & 2.4375 & 11.5 & 2.5 & 0 \\
\hline 1 & 1 & 1.71875 & 13.5 & 2 & 0 \\
\hline 2 & 2 & 1.859375 & 15 & 1.5 & 0 \\
\hline 3 & 3 & 2.4296875 & 17.5 & 2.5 & 0 \\
\hline 2 & 2 & 2.21484375 & 20 & 2.5 & 0 \\
\hline 1 & 1 & 1.607421875 & 21.5 & 1.5 & 0 \\
\hline 3 & 3 & 2.303710938 & 23.5 & 2 & 0 \\
\hline 1 & 1 & 1.651855469 & 25.5 & 2 & 0 \\
\hline 2 & 2 & 1.825927734 & 27 & 1.5 & 0 \\
\hline 3 & 3 & 2.412963867 & 29.5 & 2.5 & 2.5 \\
\hline 2 & 2 & 2.206481934 & 32 & & \\
\hline
\end{tabular}

\subsection{Road Bumps Detection}

By tracking the accelerometer reads and doing the above calculations in Table 2, the value of "Transformed Y-Bump Factor" is joined to each read instance of accelerometer. As shown from Table 2. the value "Transformed Y-Bump Factor" it is obvious that it could have either zero or a positive value, where zero values indicate that no bump occurring at the time this read is taken, while a positive value means that we are currently passing over a bump/hole (or a part of an ongoing bump/hole) as illustrated in Figure 2.

When a bump is starting to occur the value of "Transformed Y-Bump Factor" (TYBF) changes from zero to a positive value. The amount of this value could vary according to the sharpness of the bump. For acute bumps, or holes, the value tends to be larger.

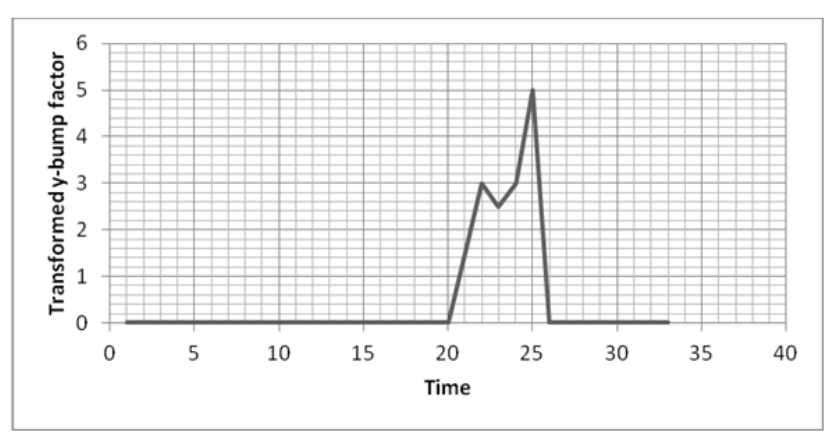

Figure 2. Transformed y-bump factor with slope elimination
Once the starting of speed bump is detected the mobile application assigned a starting point for the bump and keeps listening to the calculated consecutive reads of "Transformed Y-Bump Factor" (TYBF).

As long as this value is still positive, the application assumes we are still passing over a current bump till the value turns again to zero, then the application assigned that the current bump ended. As the experiments show, the changes (delta) between consecutive reads of $\mathrm{Y}$ axis, It happens that a zero delta occurs while the vehicle is still being crossing over the bump because the bump itself is often not regular as shown in Figure 2. between 22 and 25. Bumps often consist of two or more consecutive irregular up/down bends. While crossing over a bump, the vehicle could inverse from up bend to down bend or from down to up bend. This causes the application to pick a zero delta while monitoring the $\mathrm{Y}$ axis changes over the bump. So, a typical bump yields consecutive positive values of "Transformed Y-Bump Factor" (TYBF) with some zero values occurring between the positive values. In this case, the application doesn't understand that those zero values are not real signs for ending current bump. The Application does not mark the bump as ended for just a zero TYBF detected. The application ignores the zero values that are repeated for small number of times during bumps occurring. It still ignores those zero values until it reaches to the prespecified constant number of times before assigning that the occurring bump is ended. 
In order to assess the accuracy of our proposed model we have tested the developed application in up and down sloppy roads and resulted in acceptable results but there is a slight impact due to the fact that the captured change in the $\mathrm{Y}$ axis is not caused only by bumps but by the nature of the road itself. To exclude the effect of the slope of the road, the application has to first isolate this effect as shown in Table 3. and in Figure 3. As in the proposed model it is enhanced to be able to detect how much does the sloppy road affect the changes in the $\mathrm{Y}$ axis, as we have to realize that most of the roads are not fully horizontal with zero changes in the $\mathrm{Y}$ axis along all the road even if the road seems to be like that as shown in Table 3. We intend to detect the road Slope-Effect (SE) by first calculating the result of dividing the "Delta Y-Average" $(D A v Y)$ by "Delta XAverage" $(D A v X)$ for each accelerometer read instance.

Table 3. Transformed y-bump factor with considering Slop effect

\begin{tabular}{|c|c|c|}
\hline Delta AvgY & $\begin{array}{c}\text { Transformed } \\
\text { y-bump factor }\end{array}$ & Slope-Effect \\
\hline 0 & 0 & \\
\hline 1 & 0 & 1 \\
\hline 2.5 & 0 & 1.5 \\
\hline 2 & 0 & 0.5 \\
\hline 1.5 & 0 & 1 \\
\hline 2.5 & 0 & 1.5 \\
\hline 2 & 0 & 0.5 \\
\hline 1.5 & 0 & 1 \\
\hline 2.5 & 0 & 1.5 \\
\hline 2.5 & 0 & 1 \\
\hline 1.5 & 0 & 0.5 \\
\hline 2 & 0 & 1.5 \\
\hline 2 & 0 & 0.5 \\
\hline 1.5 & 0 & 1 \\
\hline 2.5 & 0 & 1.5 \\
\hline 2.5 & 0 & 1 \\
\hline 2 & 0 & 1 \\
\hline 2 & 0 & 1.5 \\
\hline 2.5 & 0 & \\
\hline & &
\end{tabular}

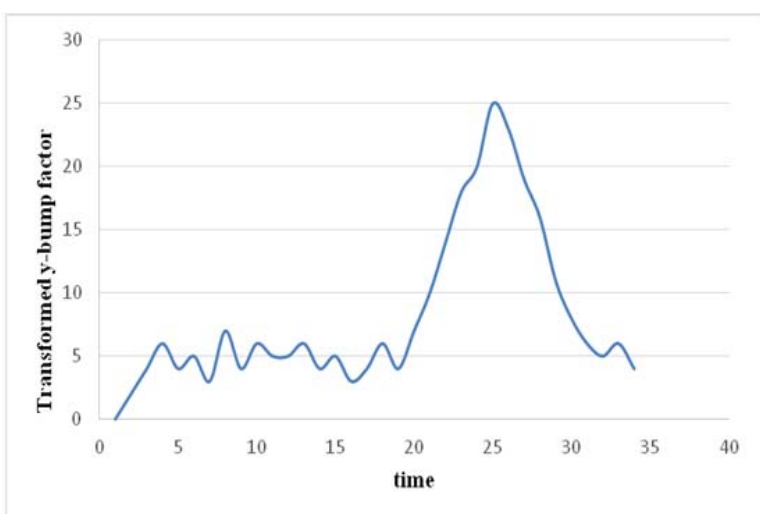

Figure 3. Up-roads and down-roads with slope-effect
Second, we calculated the average of some consecutive occurrences to find what we called slope-effect-refinement-factor. Again, by trial and error when testing the proposed model against many sloppy up/down roads, we have been convinced to perform the calculation of slope-effect-refinementfactor in regular periods basis which has been adjusted to be between 10 seconds up to 30 seconds. Finally, we involve the value of the calculated slopeeffect-refinement-factor by adding it to the "noisefactor" base constant value each time we calculate it. By adding the slope-effect-refinement-factor value to the "noise-factor", we then actually have reduced the slope-effect of the road by telling the application to tune the "noise-factor" according to the changes in the road nature as shown in Figure 4.

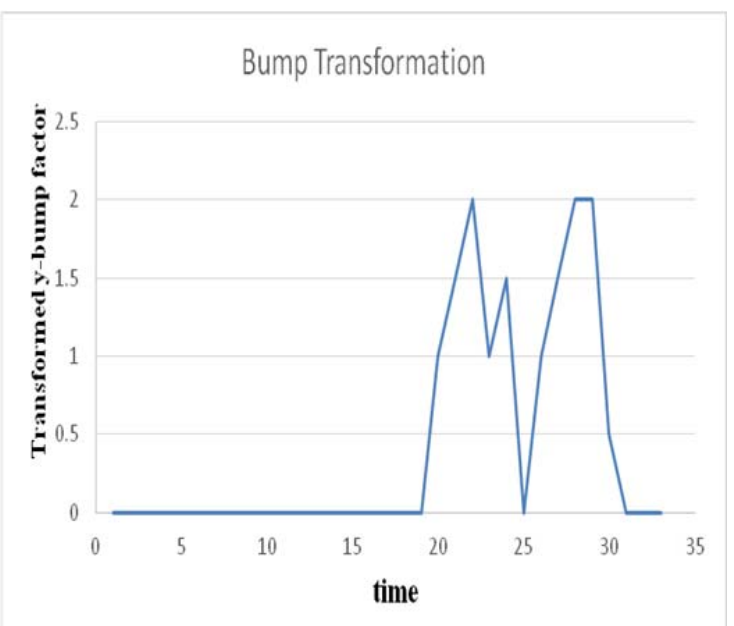

Figure 4. Transformed y-bump factor slope-effectrefinement-factor

\section{Conclusion}

The proposed model in this paper aimed to detect the road speed bumps and potholes by a mobile application through utilizing the Accelerometer and the GPS sensors embedded within the mobile phones to alert other vehicles and city authorities to keep track of the road conditions. The proposed model was implemented as an Android which is equipped with a noise filtering technique through a noisethreshold to enable the mobile application to IGNORE the results caused by phone-shaking. The proposed model introduced an approach for tracking and calculating the variations on the Y-axis of the accelerometer to detect the road speed bumps and holes. Moreover, the proposed model was tested in straight-forward road as well as in going-up roads (Climbing-up), and in going-down roads (Slopedown) and the results were quite accurate and show that the proposed model was able to detect all road speed bumps. 


\section{Acknowledgement}

The authors are grateful to the Middle East University, Amman, Jordan for the financial support granted to cover the publication fee of this research article.

\section{References}

[1]. Al-Shargabi, B., \& Sabri, O. (2017, May). Internet of Things: An exploration study of opportunities and challenges. In 2017 International Conference on Engineering \& MIS (ICEMIS) (pp. 1-4). IEEE.

[2]. Celaya-Padilla, J., Galván-Tejada, C., LópezMonteagudo, F., Alonso-González, O., Moreno-Báez, A., Martínez-Torteya, A., ... \& Gamboa-Rosales, H. (2018). Speed bump detection using accelerometric features: a genetic algorithm approach. Sensors, 18(2), 443.

[3]. Li, X., Shu, W., Li, M., Huang, H. Y., Luo, P. E., \& Wu, M. Y. (2008). Performance evaluation of vehiclebased mobile sensor networks for traffic monitoring. IEEE transactions on vehicular technology, 58(4), 1647-1653.

[4]. Barabba, V., Huber, C., Cooke, F., Pudar, N., Smith, J., \& Paich, M. (2002). A multimethod approach for creating new business models: The General Motors OnStar project. Interfaces, 32(1), 20-34.

[5]. Silva, T. H., De Melo, P. O. V., Viana, A. C., Almeida, J. M., Salles, J., \& Loureiro, A. A. (2013, November). Traffic condition is more than colored lines on a map: characterization of waze alerts. In International Conference on Social Informatics (pp. 309-318). Springer, Cham

[6]. Kariler, M., (2017). Road Surface Quality Detection Using Accelerometer Data. Master Thesis.

[7]. Ganesan, R. K., Natesan, D. K., Raj, A., Sekar, N., \& Sundaram, N. S. (2015). An image processing approach to detect obstacles on road (No. 2015-260006). SAE Technical Paper.

[8]. Sattar, S., Li, S., \& Chapman, M. (2018). Road surface monitoring using smartphone sensors: A review. Sensors, 18(11), 38-45.

[9]. Koch, C., \& Brilakis, I. (2011). Pothole detection in asphalt pavement images. Advanced Engineering Informatics, 25(3), 507-515.

[10]. Wang, K. C. P. (2004). Automated pavement distress survey through stereovision (No. NCHRP-IDEA Project 88).

[11]. Haq, M. U. U., Ashfaque, M., Mathavan, S., Kamal, K., \& Ahmed, A. (2019). Stereo-Based 3D Reconstruction of Potholes by a Hybrid, Dense Matching Scheme. IEEE Sensors Journal, 19(10), 3807-3817.

[12]. Yan, W. Y., \& Yuan, X. X. (2018). A low-cost video-based pavement distress screening system for low-volume roads. Journal of Intelligent Transportation Systems, 22(5), 376-389.
[13]. Alhadithy, H., \& Al-Shargabi, B. (2018, October). Fuzzy rule based web service composition in cloud. In Proceedings of the First International Conference on Data Science, E-learning and Information Systems (p. 27). ACM.

[14]. Tonde, V. P., Jadhav, A., Shinde, S., Dhoka, A., \& Bablade, S. (2015). Road quality and ghats complexity analysis using Android sensors. International Journal of Advanced Research in Computer and Communication Engineering, 4(3), 101-104.

[15]. Alessandroni, G., Klopfenstein, L. C., Delpriori, S., Dromedari, M., Luchetti, G., Paolini, B., ... \& Bogliolo, A. (2014). Smartroadsense: Collaborative road surface condition monitoring. Proceedings of the UBICOMM, 210-215.

[16]. Astarita, V., Caruso, M. V., Danieli, G., Festa, D. C., Giofrè, V. P., Iuele, T., \& Vaiana, R. (2012). A mobile application for road surface quality control: UNIquALroad. Procedia-Social and Behavioral Sciences, 54, 1135-1144.

[17]. Silva, N., Shah, V., Soares, J., \& Rodrigues, H. (2018). Road anomalies detection system evaluation. Sensors, 18(7), 1984

[18]. Seraj, F., van der Zwaag, B. J., Dilo, A., Luarasi, T., \& Havinga, P. (2015). RoADS: A road pavement monitoring system for anomaly detection using smart phones. In Big data analytics in the social and ubiquitous context (pp. 128-146). Springer, Cham.

[19]. Ng, J. R., Wong, J. S., Goh, V. T., Yap, W. J., Yap, T. T. V., \& Ng, H. (2019). Identification of Road Surface Conditions using IoT Sensors and Machine Learning. In Computational Science and Technology (pp. 259-268). Springer, Singapore.

[20]. Anaissi, A., Khoa, N. L. D., Rakotoarivelo, T., Alamdari, M. M., \& Wang, Y. (2019). Smart pothole detection system using vehicle-mounted sensors and machine learning. Journal of Civil Structural Health Monitoring, 9(1), 91-102.

[21]. Vachan, B. R., \& Mishra, S. (2019, April). A User Monitoring Road Traffic Information Collection Using SUMO and Scheme for Road Surveillance with Deep Mind Analytics and Human Behavior Tracking. In 2019 IEEE 4th International Conference on Cloud Computing and Big Data Analysis (ICCCBDA) (pp. 274-278). IEEE.

[22]. Allouch, A., Koubâa, A., Abbes, T., \& Ammar, A. (2017). Roadsense: Smartphone application to estimate road conditions using accelerometer and gyroscope. IEEE Sensors Journal, 17(13), 4231-4238.

[23]. Varma, V. S. K. P., Adarsh, S., Ramachandran, K. I., \& Nair, B. B. (2018). Real Time Detection of Speed Hump/Bump and Distance Estimation with Deep Learning using GPU and ZED Stereo Camera. Procedia computer science, 143, 988-997. 